\title{
Future Product Lifecycle Management (PLM) - A Consideration of Informal Communication as a Key Enabler for Future Product Development
}

\author{
Andrea Denger and Wolfgang Unzeitig \\ Virtual Vehicle Research Center, Graz, Austria \\ \{andrea.denger, wolfgang. unzeitig\}@v2c2.at
}

\begin{abstract}
Doing business globally involves very complex and increasingly dynamic processes and demands a high level of flexibility and adaptability from the companies involved. In the automotive sector, the timescale for development of new vehicles has been drastically shortened, while at the same time the number of variants and derivatives has grown. Under these conditions, development processes, involving communication across different disciplines and locations need to be flexible and efficient. In its research project Future PLM, the project consortium investigates future demands for product life cycle management in terms of the way it handles roles and participation of people. The project intends to discover ways in which PLM can be made more successful in future and to pinpoint challenges that PLM will have to meet. A catalogue of requirements for PLM 2020 was drawn up on the basis of the results of interdisciplinary panels, four future scenarios, qualitative and quantitative interviews and of a sector-specific use case for the automotive industry. In this paper the focus is set on an important matrix intersection: human \& communication. As a result a concept for informal communication and its demands on a future product lifecycle management are shown together with an example on a social networking tool (e.g. Google Plus).
\end{abstract}

Keywords: Future PLM, Informal communication, Product development, Product lifecycle management, Social networking.

\section{Introduction and Motivation}

Industrial challenges in the automotive industry are more and more focused on the optimization of the development process which requires new integrated instruments of communication and collaboration. Doing business globally engages very complex and increasingly dynamic processes and demands a high level of flexibility and adaptability from the companies involved. In the automotive sector, the timescale for development of new vehicles has been drastically shortened, while at the same time the number of variants and derivatives has grown. Under these conditions development processes, sharing information across different disciplines, locations, and partners needs to be flexible and efficient. There is the need to ensure that knowledge about the product development and the manufacturing processes is made available in the 
organization at the time and place when it is relevant. External influences which require information hierarchies and formalized procedures have to be loosened up and transformed into collaboration systems that reflect natural communication behavior and which people are willing to accept. People working in complex systems have to make decisions with long-term consequences and under pressure. The challenge facing a PLM system is how to provide the people with suitable support for their decisions.

In its research project Future PLM, the Virtual Vehicle Research Center investigates future demands for product life cycle management in terms of the way it handles roles and participation of people. The project intends to discover ways in which PLM can be made more successful in future and to pinpoint challenges that PLM will have to meet. The results presented here have been worked out on the basis of contributions from all project partners.

\section{Definition of Topic and Term}

Product Lifecycle Management in the scope of this paper is seen as a strategic concept used for managing intellectual properties of a product over the entire lifecycle [1]. However, in order to integrate a holistic PLM strategy, the improvement of processes and methods within the product development process is a necessity. Challenges in future product development include:

- managing the entire lifecycle of a product

- collaboration between different disciplines and cultures

- globally distributed development locations

- integration of customers and suppliers

A catalogue of requirements for PLM 2020 was drawn up on the basis of the results of interdisciplinary panels, four derived future scenarios on product development in 2020, qualitative and quantitative interviews in automotive companies and a sectorspecific use case for the automotive industry [1]. For a better understanding, the catalogue of requirements is inset in a matrix with specific levels versus topics (an excerpt is shown in Table 1). The levels are structured into human, organization, method, process, and IT system. A selection of topics is communication, interdisciplinarity and management of data, information and knowledge. In the catalogue of requirements the previously collected ideas and suggested solutions are clarified and documented along with their chain of effects.

In this paper the focus is set on an important matrix intersection: human \& communication. An essential issue of this intersection is informal communication during the early stages of product development. Informal communication is defined as interaction between participants without guidance, enabling people to interact in any desired way. The participants decide for whatever reason to engage each other. One aspect is to outline domain and company interdependencies that offer potential to improve cooperation and collaboration. Fish [2] already stated in 1990 that informal communication is required for coordination and that without informal communication much collaboration could not be created or would fail. 
Table 1. Matrix with specific levels compared with an extract of the topics

\begin{tabular}{|l|l|l|l|l|l|}
\hline $\begin{array}{l}\text { Levels / } \\
\text { Topics (sample) }\end{array}$ & Human & Organization & Method & Process & IT-systems \\
\hline $\begin{array}{l}\text { Socio-technical impacts } \\
\text { on future workplace }\end{array}$ & requirements & requirements & requirements & requirements & requirements \\
\hline $\begin{array}{l}\text { Communication in prod- } \\
\text { uct development \& cross- } \\
\text { domain collaboration }\end{array}$ & requirements & requirements & requirements & requirements & requirements \\
\hline $\begin{array}{l}\text { Intercultural cooperation } \\
\text { sibility }\end{array}$ & requirements & requirements & requirements & requirements & requirements \\
\hline $\begin{array}{l}\text { Older employees in } \\
\text { businesses }\end{array}$ & requirements & requirements & requirements & requirements & requirements \\
\hline $\begin{array}{l}\text { Co-Creation from the } \\
\text { consumer perspective }\end{array}$ & requirements & requirements \\
\hline $\begin{array}{l}\text { Sharing of knowledge } \\
\text { and experience }\end{array}$ & requirements & requirements & requirements & requirements & requirements \\
\hline
\end{tabular}

According to Golas [3] a company represents an open and targeted social system in its environment. Humans in the field of automotive industry are working in complex socio-technical systems. No matter how much technically dominated this operational environment is - the creative, social and individual facets of people remain very important. They are working with complex technical systems in a social system - such systems are characterized on the one hand by routines and on the other hand by exceptional situations or crises. These situations require e.g. that people make decisions under time pressure and high risk because of diffuse information or find new innovative solutions within very limited time. Therefore people represent the significant potential in a company and have to get major support in communication and collaboration to achieve the corporate goals. In accordance with Golovatchev and Budde [4] complexity of relations in development and manufacturing industry requires collaborative PLM processes with defined interfaces, i.e. there should be an informational architecture that supports collaboration.

As a result a concept for informal communication and its demands on a future product lifecycle management are shown together with an example on a social networking tool (e.g. Google+ [5]). Based on the developed communication model a method is shown how to improve the teamwork inside companies step by step in relation to global trends in cooperation, which will be faced in the next 10 years. 


\section{Definition of Method}

At the beginning of the research work the question dominated, how people communicate in the environment of product development und what expectations towards technology people have. Based on this concern the industrial as-is situation has been elaborated with a series of qualitative interviews. In such interviews data are obtained from a relatively small group of people. This differentiates it from quantitative analyzed data having a statistical significance. The interviewees are working in different organizational positions - design engineer, group manager and head of department and in all phases of product development as well [6]. Out of these interviews core messages were extracted.

First the target function of the framework was determined: collaboration needs informal communication to be efficient. In the next step additional functions have been identified besides this target function. Considering their necessary input and the gained output of each function a logical separation and sequence was modeled. This was the origin of the supporting functions and core functions. Together the whole model of the framework for informal communication was established. In this paper 'framework' is defined as a boundary of a system in which subsystems interact with each other.

\section{Framework of Informal Communication (FOIC)}

The framework of informal communication (FOIC) combines functionality which supports informal communication within distributed companies.

The situation of product development in the automobile industry has to be considered: Engineers are dealing with a situation of distributed development - distributed to different specialist domains, to different organizations, to different countries, cultures and time zones. On the other hand engineers are forced to fulfill market requirements on rapidly increasing variants and derivates combined with decreasing development periods. Informal communication is an essential precondition for coordination and collaboration [2] as already mentioned before. Especially in the described challenging environment this part of communication should not be neglected.

The framework of informal communication establishes eight different functions, in which support of informal communication is prior. Each function focuses on an individual benefit. Nevertheless there exist dependencies of each function on other functions inside the framework.

- Developing culture (building culture of dialogue)

- Building trust

- Support of relationship development

- Encourage of information exchange

- Support of work related tasks

- Support of coordination

- Support of team building

- Support of collaboration 
Following Parts of the framework have already been identified as main task of informal communication by Whittaker [7].

- The execution of work related tasks

- Coordination of group activities

- Transmission of office culture

- Social functions, e.g. team building

This input was the starting point of the development of FOIC. The target function of the framework is to support collaboration. Besides the companies need to develop culture and to build trust within their teams as a supporting function. The core functions are a logical sequence of the remaining parts. Is it able to give an answer to the question how collaboration can be developed in the best way? [8] This question will be discussed in the next chapter.

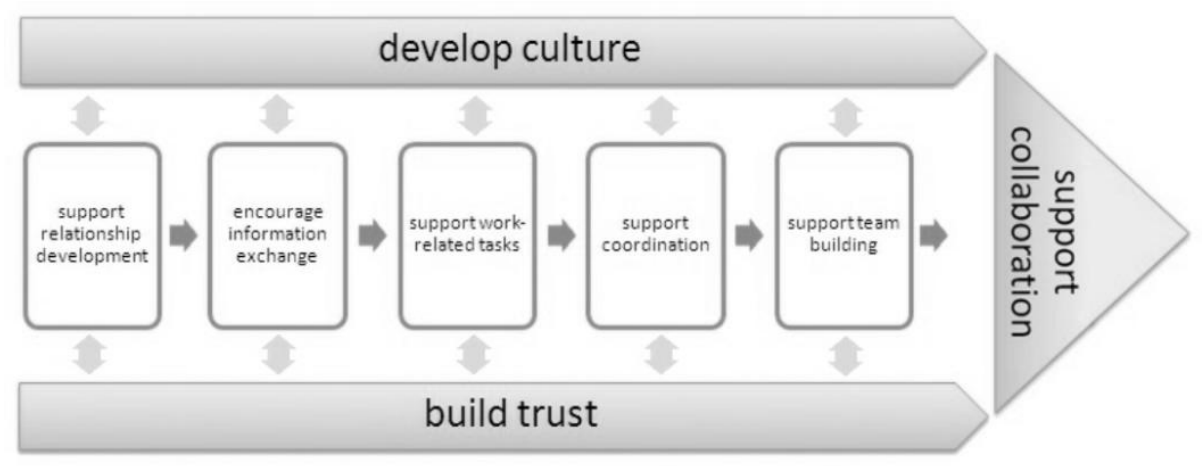

Fig. 1. Framework of Informal Communication: The framework is divided into three areas: supporting function (develop culture and build trust), core function (support relationship development, encourage information exchange, support work-related tasks, support coordination, support team building) and target function (support collaboration)

\subsection{The Three Classifications of FOIC}

\section{Target Function}

Supporting collaboration, which is defined as working together of minimum two people to achieve a common goal, is defined as the target function in the framework [9]. The involved people are dependent on each other. Because of this dependency they need to connect to each other in a most efficient way. Getting in contact is highly related to communication and especially to informal communication. With a gradual merging of a team described in the FOIC model there is high potential to generate flexible and result-oriented collaboration.

\section{Supporting Functions}

The first function of the supporting functions is to build a culture of dialogue. The way people interact with each other, i.e. by commonly used vocabulary or a dress code is defined by the enterprise culture. An enterprise culture is formed over time 
and depends on the behavior of everyone in the organization. The culture of dialogue influences all other functions as it defines the informal behavior between employees. It is necessary to recognize and accept all cultures and behavior patterns in order to cooperate successfully [10]. Especially in virtual teams everyone has to deal with the challenge to consider all the different cultures and build up a new culture of dialogue together. [7]

The second support function is to build trust. Trust is defined as the act of relying on the action of another person [11]. Trust between two people is usually based on shared experiences. This means trust is existent when one party is convinced that the other party is qualified to accomplish a task. [7]. Trust is generated through perpetual interaction and boosts efficiency in project teams [12]. It has a crucial impact on all other functions of the discussed framework and can be increased by sharing information.

Both supporting functions have a major influence on all corresponding core functions. Without trust and culture of dialogue it will be very difficult to share information, help each other in specific work tasks and finally merge to a team.

\section{Core Functions}

The core function itself consists of five consecutive parts with their overall target to support collaboration. At the beginning of collaborative working there is a need that relationship must be build within the team. In this framework relationship is defined as a network of contacts. The collaborators are connected and know of the existence of each other. Once these connections exist, assistance can be offered within the team as the first step. [13]

When the collaborators are aware of the expertise of each other, the basis has been built to exchange information. The closer they are, the easier the information can be exchanged [8]. Especially weak ties [13] need to be supported. The act of exchanging information is primarily accomplished by conversations although different possibilities of information exchange are available. For example in corporate social networking environments or corporate blogs people share information without necessary starting a conversation. [7]

Now the team members know who has knowledge about special problems and how to share information with the knowledge keeper. As a next step they are able to combine this information with their actual work tasks and gain help inside their network for coping with current challenges. At this point the information in the network is target-oriented to specific work tasks.

From this point on, more than one person is working on the same problem. This is the origin of coordination. As soon as several people are working together, as soon there exist interfaces and shared problems which are needed to coordinate the coworkers to get results efficiently. Everyone needs to work on the overall target without neglecting the specific target of the work task. To keep all the members on trace it is necessary to establish a coordination system or to support the members in establishing such a system.

As a result the teambuilding itself is supported: There is already interaction, information flow and coordination going on. The cooperators know each other, they know about their strengths, how to share information, provide each other help and have a coordination system. All these activities require the relation not only to work on a professional level but especially on a social and emotional level. 


\subsection{Strengths and Weaknesses of the FOIC Functions within the Automobile Product Development}

The following example of the automotive product development and its approach of work distribution shows: Thinking of the diversified team all around the world the main focus of informal communication should be supporting the collaboration itself.

Starting a project with the task to develop a new car with distributed engineering centers there will be members inside the team who might know each other and others who don't. Some may have experiences with some of the involved culture and some might be complete new in a multicultural project. So the project management needs to bring the people together, get them into relationship and encourage communication inside the team according to the FOIC. This getting together will have formal and informal components. Since here the informal components are especially considered, there is the need to implicate trust and a common culture from the very beginning. Therefore it is at the start where the basis for a trustful communication is built. Then the next parts of the discussed framework will follow: the project team will exchange information, the experts are well known, assistance is provided for specific work task with an efficient coordination within the team. Now there are solutions for requirements engineering, for data exchange and for design and validation etc. but finally the project team needs to face with the opportunities of informal communication and how to involve it into product development. Still it is hard to neglect that it is a challenge to measure the success of informal communication and to guide an efficient player inside collaboration teams. These tasks need to be further investigated and their results proven.

\subsection{Application of FOIC in Google+}

Which tool and features on the free market is able to support the functions of the developed framework for informal communication (FOIC)? In this chapter the focus will be on the features of Google+ and how these features can go along with the functions of FOIC. Google+ is a free social network tool based on the idea to create groups for your connected people, with whom you can share thoughts, links and pictures. [5]

As a first step the feature "Hangout" has the possibility to encourage the culture development inside enterprises. It gives the collaborators the possibility to get easily in contact with each other, develop their communication language, share ideas and discuss them with others. This already shows the second power of "Hangout". It animates the users to exchange their information.

Secondly the feature "Circles" provides the possibility to combine the linked collaborators to special interest groups, which can be connected at once. You get an overview of your team members; differ between people with whom you are in near contact and people with whom you just have weak ties. Anyway the contact won't get lost and can be restarted at any time.

Thirdly Google+ gives the possibility of "Location". Anyone can tell the other connected people where they are at the moment. This information is a key factor for the support of work-related tasks combined with culture. If you have the information about the cultural background of your collaborators you are able to consider these cultural differences in your communication work and develop ideas for current task problems more efficiently. 


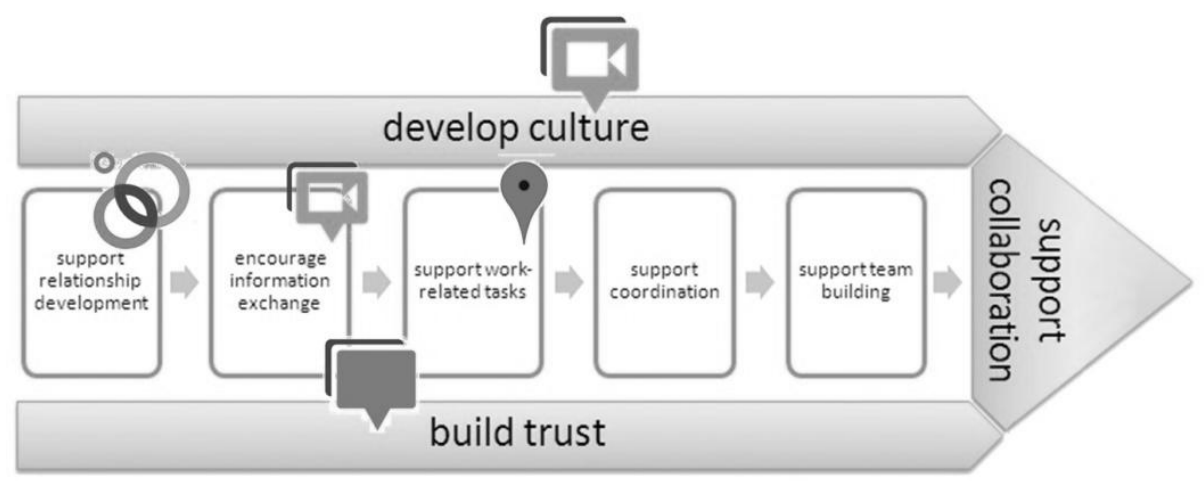

Fig. 2. Google+ solutions for Framework of Informal Communication: Google+ is supporting following parts of FOIC: Support relationship development with "Circles", encourage information exchange with "Hangout", support work-related tasks combined with develop culture with "Location" and develop culture with "Hangout" again. A tool feature is missing to support coordination, support of teambuilding and support collaboration itself

Finally there are some parts not yet supported by Google+. Features for coordination and team building would give this tool a powerful capability to foster collaboration with special support for informal communication. The discussed framework requires additional tools for these functions.

\section{The Challenge to Realize FOIC}

The discussed framework divides the field in several parts, which can be supported with specific tools. Nevertheless not only tools are the solution. Still the organization of the collaboration team, the organizational processes and last but not least the human itself need to be considered. Since trust and corporate culture is mainly influenced by the people there are long term steps and assistance necessary to create and maintain a positive and creative climate within the team.

On the other hand software solutions support especially the core functions. There can be building blocks implemented step by step. Still they should have the common target of tools, fitting together and leading to an accepted, user- and solution-oriented information flow and cooperation process. Obviously an important starting point is supporting relationship development. Only with relationships - virtually or in real life -people will communicate and gradually align their discussion to the target of your project and enterprise.

\section{Conclusion}

With the framework FOIC enterprises are in a position to support and work on specific functions of informal communication. A combination of all functions enables a supported collaboration. Without only one of these functions, collaboration will be 
more difficult to start und less effective. If there is a team of collaborators who are physically close to each other, informal communication will happen anyway and the framework will rise accompanied by empathic leadership. On the other hand, if there is the situation of distributed work - to different location, different knowledge domains, different cultures, etc. - the enterprise do have the possibility to use the power of the rising social networks within their borders. Software solutions can stimulate the informal communication and lead to a target oriented collaboration.

This study has its limitations. In this paper the theoretical background of the framework and an application example with Google+ was presented. However an enterprise-specific solution with long time analysis of the strengths and weaknesses is a next step to discuss the ability of the framework.

The paper has highlighted many issues regarding collaboration in combination with informal communication. Still more work will be required to examine the details occurring in different types of application with an international background.

Acknowledgements. The authors would like to acknowledge the financial support of "COMET K2-Research Centres for Excellent Technologies Programme" of the Austrian Federal Ministry for Transport, Innovation and Technology (BMVIT), the Austrian Federal Ministry of Economy, Family and Youth (BMWFJ), the Austrian Research Promotion Agency (FFG), the Province of Styria and the Styrian Business Promotion Agency (SFG). We would furthermore like to express our thanks to our supporting industrial and scientific project partners, namely "AVL List GmbH", "BMW AG", "CSC Computer Science Consulting Austria GmbH", "MAGNA STEYR Fahrzeugtechnik AG \& Co KG", Vienna University of Technology, University of Kaiserslautern, and to the Graz University of Technology.

\section{References}

1. Schmeja, M., Denger, A.: FuturePLM - Ansätze für ein mitarbeiterzentriertes PLM. In: Proceedings of Product Life Live, 2011, pp. 129-136 (2011)

2. Fish, R.S., Root, R.W., Chalfonte, B.L.: Informal Communication in Organiziations: Form, Function and Technology. In: Oskamp, S., Spacapan, S. (eds.) Human Reactions to Technology: The Claremont Symposium on Applied Social Psychology, Sage Publications, Beverly Hills (1990)

3. Golas, H.G.: Der Mitarbeiter, 9th edn. Verlag Cornelsen Lehrbuch (1997)

4. Golovatchev, J.D., Budde, O.: Next generation PLM - an integrated approach for the Product Lifecycle Management. In: Proceedings of ICCPR 2007: International Conference on Comprehensive Product Realization 2007, Beijing, China, June 18-20 (2007)

5. Google+, http://www.google.com/+/learnmore/ (accessed on January 31, 2012)

6. Denger, A., Maletz, M., Helic, D.: Social Computing: A Future Approach of Product Lifecycle Management. In: Proceedings of I-KNOW 2010: 10th International Conference on Knowledge Management and Knowledge Technologies, pp. 451-457 (2010)

7. Neubauer, H.: Human Communication and Virtual Product Development. Diploma Thesis, FH Joanneum - University of Applied Sciences, Graz (2011) 
8. Whittaker, S., Frohlich, D., Daly Jones, O.: Informal Workplace Communication: What is it like and how might we support it? In: Human Factors 1994 (1994)

9. Tellioglu, H.: Forming a Collaboration by Using Coordination Design. In: Proceedings of the 3rd International Conference on the Application of Digital Information and Web Technologies (ICADIWT 2010), Istanbul (2010)

10. Heussen, H.: Produkt und Mensch im interkulturellen Chaoswirbel - Wie steuert man den Durchblick? In: 3rd FuturePLMInterdisciplinary Exchange of Experience, Graz (2011)

11. Lo, S., Lie, T.: Selection of communication technologies - A perspective based on information richness theory and trust. Technovation 28(3), 146-153 (2008)

12. Iacono, C.S., Weisband, S.: Developing trust in virtual teams. In: Proceedings of the Thirtieth Hawaii International Conference on System Science, Hawaii (1997)

13. McAfee, A.: How to Hit the Enterprise 2.0 Bullseye (2007),

http://andrewmcafee.org/2007/11/how_to_hit_the_enterprise_20 _bullseye/ (accessed on January 31, 2012) 